\title{
Influence of spatial environment on maze learning in an African mole-rat
}

\author{
Lydia du Toit ${ }^{1,2}$, Nigel C. Bennett ${ }^{3}$, Alecia Nickless ${ }^{1,4}$ and Martin J. Whiting ${ }^{1,5}$
}

\begin{abstract}
${ }^{1}$ School of Animal, Plant and Environmental Sciences, University of the Witwatersrand, Private Bag 3, Wits 2050, South Africa

${ }^{2}$ Obesity Research and Management, University of Alberta, Edmonton T6G 2R3, Canada (present address)

${ }^{3}$ Mammal Research Institute, Department of Zoology and Entomology, University of Pretoria, Pretoria 0001, South Africa

${ }^{4}$ Natural Resources and the Environment, Council for Scientific and Industrial Research, PO Box 395, Pretoria 0001, South Africa (present address)

${ }^{5}$ and, Department of Biological Sciences, Division of Brain, Behaviour and Evolution, Macquarie University, Sydney 2109, Australia (present address)
\end{abstract}

Corresponding author: Martin Whiting: martin.whiting@mq.edu.au

RH: Cognition in mole-rats

\begin{abstract}
In subterranean species where excavation is energetically expensive, efficient spatial navigation is vital to reducing the costs of locating important resources such as food and mates. While spatial navigational ability is positively correlated with sociality in subterranean mammals, we have a less clear understanding of the role of habitat complexity on navigational ability. We tested spatial navigational ability and memory in 12-18 month captive Natal mole-rats (Cryptomys hottentotus natalensis) maintained in a simple environment with no environmental enrichment and newly captured wild individuals from natural, complex burrow systems. In maze trials, mole-rats captured freshly from the wild
\end{abstract}


made significantly fewer navigational errors, were more likely to successfully navigate the maze, travelled shorter distances and as a consequence, completed the maze in less time. Male mole-rats from both experimental treatments were more likely to complete the maze than females. Memory retention of the maze was tested on day two, seven, 30 and 60 respectively. The results were variable, although both groups showed a significant memory retention 60 days after testing. Our results highlight the potential importance of the environment (microhabitat complexity) on spatial cognitive performance in mole-rats.

Keywords mole-rat, cognition, memory, learning, navigation

\section{Introduction}

Subterranean animals are not an obvious choice for spatial memory experiments because their visual systems are often regressed ( $\mathrm{N}^{`}$ emec et al. 2007) and compared to surface dwelling animals, they have a limited range of cues for navigating their environment (Burda et al. 1990a; Sherry 1998). Efficient spatial orientation and memory is, however, critical for fossorial species in their search for food patches or mates, as underground locomotion and excavation is comparatively expensive (Lovegrove 1989; Vleck 1979, 1981; Zelová et al. 2010). In the absence of light, subterranean species use olfactory (Heth et al. 2002) and somatosensory stimuli (Kimchi and Terkel 2004b), as well as the earth's magnetic field (Burda et al. 1990b; Kimchi and Terkel 2004a) and path integration (Kimchi and Terkel 2002), to orientate themselves. Not surprisingly, these species possess remarkable abilities both in spatial orientation and memory (Costanzo et al. 2009; Kimchi and Terkel 2002, 2003). For example, blind Spalax mole-rats perform better in learning and memorizing a complex maze than both laboratory rats and Levant voles (Kimchi and Terkel 2001). 
African mole-rats of the family Bathyergidae are subterranean rodents that display a striking spectrum of sociality (Skinner and Smithers 1990). The geometry of the tunnels in which mole-rats live is a function of the level of sociality they display, with solitary species inhabiting simpler tunnel systems and more social species inhabiting a complex labyrinth of tunnels (Le Comber et al. 2002; Sichilima et al. 2008). Mole-rats are therefore a model species group with which to examine the role of the environment on spatial cognition. Recently, Costanzo et al. (2009) demonstrated that the eusocial Damaraland mole-rat (Fukomys damarensis) performs better at spatial memory tasks than the solitary Cape molerat (Georychus capensis). It therefore appears that a more complex spatial environment necessitates a better spatial memory in order for the animal to effectively and inexpensively navigate its way around its home range. Nevertheless, we need tests of how environmental complexity might affect spatial cognition within a species. This has been well studied in laboratory rats (Harris et al. 2009), but is currently poorly understood for wild rodents that navigate complex environments.

Another key issue in spatial cognition studies is the influence of sex. An increasing number of studies have found that males have better spatial cognitive ability than females in spatial tasks such as maze completion (Perdue et al. 2011) in part because of ranging behaviour. During the breeding season, males of many species increase their home range in their quest to locate multiple females (range size hypothesis, Gaulin and Fitzgerald 1986) and this presumably either stimulates cognitive ability to a greater degree than in females and/or provides a selective pressure for greater spatial cognitive ability in males. While males of many species have greater spatial cognitive ability and learning retention, this is not always the case. For example, in two species of subterranean mole-rats: the eusocial Damaraland (Fukomys damarensis) and solitary Cape mole-rat (Georychus capensis), males and females performed equally well in spatial and memory retention tasks (Costanzo et al. 2009). 
Similarly, monogamous voles show no sex difference in spatial cognitive ability while males have better spatial cognition in a promiscuous species (Gaulin and FitzGerald 1989). We therefore need to test more species with varying ecological requirements to better understand sex differences in spatial cognition.

We used the Natal mole-rat (Cryptomys hottentotus natalensis) as a model to test whether the superior spatial navigational ability displayed by social mole-rats is related to the complexity of the tunnel systems they inhabit (Hickman 1979b). In the wild, mole-rat tunnel systems continually change as colony members expand the tunnels through their foraging efforts. Conversely, captive mole-rats are maintained in simple rectangular containers and are provided with food by their caregivers. They lack the opportunity or need to enrich their spatial environment through the digging of a tunnel system. Captive animals therefore represent a unique opportunity to study the influence of habitat complexity on spatial navigational ability. We hypothesised that captive animals will have a decreased ability to navigate a complex maze when compared to conspecifics recently captured from the wild. We also asked whether there is a difference in spatial cognitive ability between males and females.

\section{Methods}

Study animals

We collected 14 male and ten female adult Natal mole-rats (Cryptomys hottentotus natalensis) from Glengarry Park in KwaZulu-Natal Province, South Africa (29¹9’24’S, $29^{\circ} 42^{\prime} 55^{\prime \prime} \mathrm{E}$ ) in July 2005 . Individual mole-rats were trapped using modified Hickman livetraps baited with sweet potato (Hickman 1979a). Additionally, 12 male and 12 female molerats, originating from the same area/population but collected in 2004, were selected from a 
single existing laboratory population (range in captivity: 16-18 months). The animals were of indeterminate age, however, none were visibly juvenile or elderly. Subordinate animals belonging to sub-species in the Cryptomys hottentotus group typically remain physically small well into adulthood (Skinner \& Smithers, 1990). According to Oosthuizen (2007), female Natal mole-rats range between $47-127 \mathrm{~g}$ and males between 40 - $162 \mathrm{~g}$. Therefore, the large range of weights seen in the study sample (56.9 - $164.9 \mathrm{~g}$ for field mole-rats, and $88.2-162.4 \mathrm{~g}$ for lab mole-rats) are within the normal range of variation reported in the literature. None of the animals used were born in captivity and all animals were sexually mature. Animals were housed in plastic containers (colonies 100 x 50 x $50 \mathrm{~cm}$; single animals $50 \times 30 \times 30 \mathrm{~cm})$ in climate controlled rooms $\left(25 \pm 1^{\circ} \mathrm{C}\right)$ at the University of Pretoria. A reversed light regime of 12 hours dark: 12 hours light was enforced for three weeks prior to commencement of the experiment in order to switch the activity patterns of the animals from nocturnal to diurnal (Hart et al. 2004). Untreated wood shavings were available as nesting material. The mole-rats were fed fresh vegetables ad libitum, from which they obtained sufficient water, since they do not drink.

Experimental groups and maze

Freshly caught adult wild mole-rats $(n=24)$ were compared to long-term laboratory animals $(n=24)$ to determine whether differences in their spatial environments (complex vs. simple) affect their ability to learn and remember spatial tasks. We constructed a complex maze, with six choice points and one correct path leading to a food reward at the end of the maze opposite to the entrance (Fig. 1; Kimchi and Terkel 2001). The maze covered an area of 100 $\mathrm{x} 120 \mathrm{~cm}$, the floor consisted of a plywood board covered with vinyl sheeting and the maze itself was constructed from dark opaque Perspex sheeting with a transparent Perspex lid. The tunnels of the maze pathway were $10 \mathrm{~cm}$ wide and $10 \mathrm{~cm}$ high. The entrance and exit to the 
maze were each fitted with a plastic tube $(25 \mathrm{~cm}$ long, $8 \mathrm{~cm}$ diameter $)$ with a removable door. A food reward was placed in the tube fitted to the maze exit.

At the completion of each trial the floor, the roof and walls of the maze were cleaned with 50\% ethanol (Costanzo et al. 2009; Kimchi and Terkel 2001). The experiment was performed in a room with no external windows and lighting was provided by a single red light $(60 \mathrm{~W})$ positioned above the maze. All trials were recorded by a single observer tracing the path of the animal on a diagram of the maze and recording the time taken to complete the maze. We also videoed all trials.

\section{Learning experiment}

The test procedure, adapted from Kimchi and Terkel (2001), was the same for all the animals. One day prior to the trial the animal was removed from its home cage and housed individually in a plastic container $(50 \times 30 \times 30 \mathrm{~cm})$. We restricted food intake until completion of either the three-day learning experiment or the memory test because food deprivation has been shown to increase motivation to explore (Kimchi and Terkel 2001). A trial began when the animal entered the maze, and was terminated when the animal reached the maze exit, or after a maximum of 20 minutes if it failed to complete the maze. Animals that successfully completed the maze were rewarded with a small piece of sweet potato $(0.5$ $\mathrm{cm}^{3}$ ). At the end of each trial the animal was returned to the plastic container, and after a complete set of trials it was returned to its home cage. The experiments were conducted between 9:00 - 16:00h. Three parameters were recorded: 1) time required to correctly complete the path in the maze; 2) number of errors made; and 3) total distance travelled during each trial. 
Each animal performed five trials daily for three consecutive days and no more than four animals were tested per day. At the end of each day the animals were provided with a small amount of food. The animals were returned to their home cages and received food $a d$ libitum at the end of the three-day period.

Memory (retention) experiment

To determine how well the animals retained the maze learning, each animal was tested on its ability to navigate the maze only once at either $2,7,30$ or 60 days from the end of the learning experiment. Six animals randomly selected from each treatment were tested for each time period. The groups consisted of three males and three females, or four males and two females (two and seven day groups for wild mole-rats and seven day group for lab mole-rats). Mole-rats were housed individually and food restricted one day prior to the trial, and returned to their home cages and regular feeding cycle at the end of the single-day experiment.

Data analysis

Since the experiment concerned the time period until a certain event, where the event in this case was the completion of the maze, a survival analysis approach was used to analyze the duration data from the experiment. In conventional survival analysis problems the normal variable of interest is the time to death, but this can be replaced by any measure of duration. The data can be considered as 'right-censored', as experiments where the mole-rat did not complete the maze within 20 minutes were terminated. We constructed a probability model that a mole-rat would complete the maze in 20 or fewer minutes using the following factors: the day on which the maze was attempted, the body mass of the mole- rat, the mole-rat's group, and the sex of the mole-rat. 
In survival analysis, this probability distribution is referred to as the hazard function, which usually refers to the rate of death at a particular point in time. In this case the hazard function describes the rate of completion of the maze over time. A common approach to obtaining the hazard function is through a non-parametric method referred to as the Cox proportional hazards model, which requires few assumptions regarding the probability distribution function (Tableman and Stahel 2009). The disadvantage of this method is that interactions between variables cannot be included, which makes this method inappropriate for this study. Alternatively, the hazard function can be modelled as a generalized linear model, but in this case a density function needs to be chosen to describe the hazard function (Crawley 2005). The simplest choice is the exponential distribution, which requires only one estimated parameter, but this assumes that the probability of completing the maze remains constant throughout the duration of the experiment. A more flexible density function is the Weibull distribution, which requires the estimation of two model parameters and can allow the rate of completing the maze to increase or decrease over time. In modelling the data, we found that the additional parameter required to fit the Weibull over the exponential distribution was significant, and therefore used the Weibull distribution. If the mole-rats showed a learning effect, we would expect a reduction in both the distance travelled and the frequency of errors as a function of the number of trials performed. To test if there was a decrease in the distance and number of errors from the first day of the experiment to the third day, we used conventional linear regressions, including 'group', sex and body mass as additional predictors.

Results

During the first day of the acquisition phase $83 \%$ (99 of 120 runs) of all wild caught animals completed the maze within the 20 min time limit compared to $81 \%$ (97 of 120 runs) on the 
second day, and $90 \%$ (108 of 120 runs) on the third day. Similarly, during the first day of the acquisition phase $81 \%$ (97 of 120 runs) of all laboratory animals completed the maze within the 20 min time limit compared to $74 \%$ ( 89 of 120 runs) on the second day, and $73 \%$ ( 88 of 120 runs) on the third day.

In modelling the hazard function of the time to completion of the maze, we considered all combinations of the four variables of interest, up to the fourth level of interaction. Models were then selected based on the significance ( $5 \%$ level of significance) of the terms in the model and the results of the Deviance tests, which compares the current model to the null model and maximal model (model with all possible combinations of variables). The most parsimonious model, containing only those interaction terms which were significant, was the model fitted with three way interactions between day, sex and body mass, between day, sex and group, and between group, sex and body mass (Table 1). This model fit as well as the maximal model (Deviance test: $P=0.31$ ), and fitted significantly better than the null model (Deviance test: $P<0.0001)$.

A plot of the hazard function (Fig. 2) shows that the rate at which mole-rats completed the maze increased with time, for all groups. However, freshly caught field molerats completed the maze at a significantly faster rate than lab mole-rats (Table 1). The difference in completion rates between field and lab mole-rats is different for males and females, with the difference being smaller for female rats. Overall, the field mole-rates had higher completion rates compared to the lab rats (Fig. 2).

The coefficients estimated for the model (Table 1) indicate that the effect of body mass on the rate at which the maze was completed depended on sex, group and day. For female mole-rats in the field group, the rate of completing the maze tended to decrease with increasing body mass, with the opposite being true for male field mole-rats. For lab mole- 
rats, increasing body mass of female rats tended to result in increased rates of completion, while for males increased body mass resulted in decreased rates of completion.

An examination of the p-values of the terms in the linear model for distance show that the day on which the trial was carried out did not have a significant effect (Table 2), unless in interaction with group, sex and body mass. The fact that the main effect for day is not significant, does not imply that the day on which the trial was performed is not important. The importance of day comes through in the interaction effects, which shows that once these other effects are accounted for, then there is a significant difference between the distance travelled on these days. If group, sex and body mass were not accounted for, then the effect of the day on which the trial was carried out would be lost. Box plots of the distance data from the learning experiment (Fig. 3) illustrate that day does not have any noticeable effect on the distance travelled, except in the case of lab females where the median distance travelled, in fact, increased from day 1 to day 3. Furthermore, the plot shows that the lab group had higher median distances compared to the field group, and this difference was enhanced for heavier mole-rats, as indicated by the positive and significant coefficient of the lab group and weight interaction term in the model (Table 2).

The estimated linear model for the number of errors shows that, as for the distance model, day was not significant unless in interaction terms with other predictors (Table 3). The number of errors made during the course of the maze was significantly affected by the molerat's sex, group, and body mass. The strongest trend in the data was that lab mole-rats tended to perform more errors compared to field mole-rats. Box plots of the number of errors (Fig. 4) show no obvious trend over time within any of the groups.

Based on the data collected during the retention trials, a hazard function was estimated for the time to complete the maze, including the time between trials as an additional predictor. In the retention trials, there was a significant effect of time between 
trials on the performance of mole-rats in the maze (Table 4). As expected, mole-rats that returned after 60 days showed the lowest retention, with approximately $60 \%$ of mole-rats not completing the maze within the 20 minute period in this group (Fig. 5). The results for molerats returning to the maze after two, seven and 30 days are not as clear. In the case of the lab mole-rats, the retention of maze information after a two day return was approximately the same as that for 60 days (Fig. 5). The lab mole-rats also show a higher retention of maze information after 30 days compared to 7 days. In the case of the field mole-rats, retention of maze information by the mole-rats completing the maze appears to be similar after 2, 7 and 30 days return, and with a much lower retention after 60 days. The generalized linear model of the hazard function shows that there are significant interactions between the number of days between maze trials, sex, group, and body mass, with significant two-way interactions between days between maze trials and sex, days between maze trials and group, and sex and body mass (Table 4). The distance travelled and the number of errors made by the mole-rats during the course of the maze was not significantly affected by the time interval between trials. The only significant predictor of the distance travelled or the number of errors made was the mass of the mole-rat, with the distance travelled and number of errors increasing with the mass of the mole-rat (estimated coefficients of 50.99 and 0.573 for the models of distance and number of errors respectively, both significant at the $5 \%$ level).

\section{Discussion}

We hypothesized that freshly captured wild mole-rats would perform better at spatial tasks than their captive counterparts, and the results from this study confirmed our expectations. The rate of successful completion of the maze increased with time for both groups, however, field mole-rats showed higher overall rates of completion than lab mole-rats. Field mole-rats also committed fewer errors and ran shorter distances in the process of completing the route. 
The most obvious factor that sets captive mole-rats apart from wild ones was the simplicity of the artificial habitation. It is however, important to note that this is not the only potential cause for the apparent cognitive decline observed in the lab animals. Captive mole-rats could lack the motivation to explore, as they are used to food being provided. Food deprivation has been shown to heighten motivation to explore a maze (Kimchi \& Terkel, 2001), however, both groups received identical treatment prior to testing, therefore, lack of motivation probably does not play a major role here. It is possible that some other physiological or behavioural response to captivity correlates with reduced activity or reduced cognition.

Animals in captivity differ from conspecifics inhabiting their natural habitat in several key features that could influence their behaviour and cognition. Captive animals are provided with food and shelter, thereby negating the need for the animal to forage or to seek or construct a home for itself. Additionally, laboratory cages are often barren and lack most of the sensory stimuli animals living in a natural setting would be exposed to. An enriched environment has been associated with increased spatial abilities as well as use-dependent plasticity in brain morphology in several species (for reviews see Clayton 1995; Van Praag et al. 2000; but see Harris et al. 2009). Cognition has also been found to change with age, and while we could not determine the absolute age of the adult mole-rats from our two cohorts, it is possible that laboratory maintained mole-rats may have been older and as a consequence suffered slightly from a reduced cognitive ability.

Sex differences in the spatial abilities of rodents are not straight forward. Although males generally perform better than females, there are significant exceptions to this trend (for a review see Harris 1978; Halpern 1986; Gaulin and Hoffman 1988). Extrinsic (e.g. study design; Roof and Stein 1999) and intrinsic (e.g. sex differences in home range size; Gaulin and Fitzgerald 1986; 1989), exploration levels, activity levels or motivation factors (Joseph 1979; Joseph and Gallagher 1980; Van Haaren et al. 1987) have been postulated as possible 
explanations for this phenomenon. In microtine voles, spatial abilities correlate with sex only when the mating system and reproductive tactics employed by each sex are taken into consideration (Gaulin and FitzGerald, 1989). Interestingly, in the bathyergid mole-rats studied to date, no sex differences in spatial learning have been found in either solitary or social species (Costanzo et al. 2009). Our results show that for both laboratory and field mole-rats, males generally performed better than females. Home range cannot play a role here, as males and females share the same tunnel system and therefore have identical home ranges. Another possible explanation might be gender differences in dispersal rates. If subordinate males were more prone to disperse from their natal colonies than females, it may necessitate better spatial capabilities. It is currently unknown whether Natal mole-rats display sex differences in dispersal rates. However, male biased dispersal has been reported in the Damaraland mole-rat, Fukomys damarensis (Hazell et al. 2000). Natal mole-rats can now be added to the growing list of species (see Jones et al. 2003, Jozet-Alves et al. 2008 for partial reviews) that show sex differences in cognitive spatial ability including, for example, humans (reviewed in Coluccia and Louse 2004), fishes (Sovrano et al. 2003), birds (Vallortigara 1996), giant pandas (Perdue et al. 2011), and cuttlefish (Jozet-Alves et al. 2008).

In female field mole-rats and male lab mole-rats, the rate of completion decreased with increasing body mass. However, in male field and female lab mole-rats, the converse was true. In terms of the numbers of errors (see Table 3), body size had a negative effect on the ability of the animal to complete the maze, with smaller mole-rats performing better than larger mole-rats. This is counterintuitive as the tunnels of our maze were probably not of optimal size for the smaller mole-rats. Mole-rats dig their own tunnel systems, with the diameter of the tunnels closely approximating the size of their cylindrical bodies (Bennett and Faulkes 2000), while leaving space for animals to turn around in tunnels (White 2005). This not only keeps excavation costs down (Vleck 1979, 1981), but ensures close contact between 
the sensory hairs on the animal's body and the wall of the tunnel (Burda et al. 1990a; Kimchi and Terkel 2004b). Subterranean animals are very dependent on somatosensory input in their spatial orientation. They perform better in mazes that are small enough for their bodies to touch the sides of the maze. Laboratory rats on the other hand, do not show improved orientation with heightened somatosensory input, but rely more on visual cues for orientation (Kimchi and Terkel 2004b). In our study, the range of body size was reasonably broad: 57$165 \mathrm{~g}$. Smaller animals would have more difficulty staying in close contact with the walls of the maze, yet they performed better in the maze than larger animals. One possibility is that smaller individuals are more affected by food deprivation and are therefore more motivated to search for the food reward compared to larger individuals.

Interestingly, neither field nor lab mole-rats improved their maze running abilities over the course of the three day learning experiment. Although rates of completion increased for all groups, the number of errors committed and the distance travelled did not decrease with experience. Therefore, although the mole-rats remembered the food reward, they did not learn faster ways of completing the maze. This counterintuitive observation could be explained by the fact that the diameter of the tunnels in the maze were too big to optimize the somatosensory impact that subterranean animals partially depend on for navigation (Kimchi and Terkel 2004b).

In summary, we demonstrate that wild mole-rats from complex environments perform better in maze tests of spatial ability than captive mole-rats maintained in simple environments. Our study suggests a role for environmental complexity, amongst other factors, in stimulating cognitive development and activity and highlights the potential importance of an appropriate environment for providing adequate cognitive stimulation in captive animals. 
Acknowledgments This work was funded by a grant from the University of the

Witwatersrand to MJW. We thank Helen Place and Luke Duncan for assistance in the field. We also thank Clive Coen, Marietjie Froneman and Paul Odendaal for their assistance. This research was approved by the Animal Ethics Screening Committee of the University of the Witwatersrand (Clearance Certificate Number: 2005-43-4); animal collection was authorized by the KwaZulu-Natal Wildlife Permits Office. The management of Glengarry Park is thanked for permission to collect animals from their property.

\section{References}

Bennett NC, Faulkes CG (2000) African mole-rats: ecology and eusociality. Cambridge University Press, Cambridge

Brett RA, 1991. The population structure of naked mole-rat colonies. In: Sherman PW, Jarvis JUM, Alexander RD (eds) The biology of the naked mole-rat. Princeton, New Jersey, Princeton University Press, pp 97-136

Jones, C. M., Braithwaite, V. A. \& Healy, S. A. 2003 The evolution of sex differences in spatial ability. Behav. Neurosci. 117, 403-411

Burda H, Bruns V, Müller M (1990a) Sensory adaptations in subterranean mammals. In: Nevo E, Reig OA (eds) Evolution of subterranean mammals at the organismal and molecular levels. Wiley-Liss, New York pp269-293 
Burda H, Marhold S, Westenberger T, Wiltschko R, Wiltschko W (1990b) Magnetic compass orientation in the subterranean rodent Cryptomys hottentotus (Bathyergidae). Experientia 46:528-30

Clayton NS (1995) The neuroethological development of food-storing memory: a case of use it, or lose it! Behav Brain Res 70:95-102

Coluccia E, Louse G (2004) Gender differences in spatial orientation: a review J Environ Psychol 24:329-340

Costanzo MS, Bennett NC, Lutermann H (2009) Spatial learning and memory in African mole-rats: The role of sociality and sex. Physiol Behav 96:128-134

Crawley MJ (2005) Statistics: an introduction using R. John Wiley and Sons, ltd., West Sussex

Gaulin SJC, FitzGerald RW (1986) Sex differences in spatial ability: an evolutionary hypothesis and test. Am Nat 127:74-88

Gaulin SJC, FitzGerald RW (1989) Sexual selection for spatial-learning ability. Anim Behav $37: 322-331$

Gaulin SJC, Hoffman HA (1988) Evolution and development of sex differences in spatial ability. In: Betzig L, Mulder MB, Turke P (eds) Human reproduction and behavior. Cambridge University Press, New York, pp 129-151 
Halpern DF (2000) Sex differences in cognitive abilities. Third edition. Lawrence Erlbaum Associates, Mahwah, New Jersey

Harris, LJ (1978) Sex differences in spatial ability: possible environmental, genetic, and neurological factors. In: Kinsbourne, M (ed) Asymmetrical function of the brain, Cambridge University Press, New York, pp 405-522

Harris AP, D’Eath RB, Healy SD (2009) Environmental enrichment enhances spatial cognition in rats by reducing thigmotaxis (wall hugging) during testing Anim Behav 77:1459-1464

Hart L, Bennett NC, Malpaux B, Chimimba CT, Oosthuizen MK (2004) The chronobiology of the Natal mole-rat, Cryptomys hottentotus natalensis. Physiol Behav 82:563-569

Hazell RWA, Bennett NC, Jarvis JUM, GRIFFIN M (2000) Adult dispersal in the cooperatively breeding Damaraland mole-rat, Cryptomys damarensis: a case study from the Waterberg region of Namibia J Zool Lond 252:19-26

Heth G, Todrank J, Begall S, Koch R, Zilgiger Y, Nevo E, Braude SH, Burda H (2002) Odours underground: subterranean rodents may not forage "blindly". Behav Ecol Sociobiol 52:53-58

Hickman GC (1979a) A live-trap and trapping technique for fossorial mammals S Afri J Zool $14: 9-12$ 
Hickman GC (1979b) Burrow system structure of the Bathergid Cryptomys hottentotus in Natal, South Africa Saugetierkundliche 44:153-62

Jackson CR, Bennett NC (2005) Is the Natal mole-rat (Cryptomys hottentotus natalensis) a spontaneous or induced ovulator? J Mamm 86:1-6

Jarvis JUM, Bennett NC (1991) Ecology and behavior or the family Bathyergidae. In: Sherman PW, Jarvis JUM, Alexander RD (eds) Biology of the naked mole-rat. Princeton University Press, Princeton, pp 66-96

Jarvis JUM, O’Riain MJ, Bennett NC, Sherman PW (1994) Mammalian eusociality: a family affair TREE 9:47-51

Joseph R (1979) Effects of rearing and sex on maze learning and competitive exploration in rats. J Psychol 101:37-43

Joseph R, Gallagher RE (1980) Gender and early environmental influences on activity, over responsiveness, and exploration. Develop Psychobiol 13:527-544

Jozet-Alves, C., J. Modéran, L. Dickel. 2008. Sex differences in spatial cognition in an invertebrate: the cuttlefish. Proc. R. Soc. B: 274:2049-2054

Kimchi T, Terkel J (2001) Spatial learning and memory in the blind mole-rat in comparison with the laboratory rat and Levant vole Anim Behav 61:171-180 
Kimchi T, Terkel J (2002) Seeing and not seeing. Current Opinion Neurobiol 12:728-734

Kimchi T, Terkel J (2003) Detours by the blind mole-rat follow assessment of location and physical properties of underground obstacles. Anim Behav 66:885-891

Kimchi T, Terkel J (2004a) A subterranean mammal uses the magnetic compass for path integration. PNAS 101:1105-1109

Kimchi T, Terkel J (2004b) Comparison of the role of somatosensory stimuli in maze learning in a blind subterranean rodent and a sighted surface-dwelling rodent. Behavioural Brain Res 153:389-395

Le Comber SC, Spinks AC, Bennett NC, Jarvis JUM, Faulkes GC (2002) Fractal dimension of African mole-rat burrows. Canadian J Zool 80:436-441

Lovegrove BG (1989) The cost of burrowing by the social mole-rats (Bathyergidae) Cryptomys damarensis and Heterocephalus glaber: the role of soil moisture. Physiol Zool 62:449-69

N`emec P, Cvekov'a O, Burda H, Benada O, Peichl L (2007) Visual Systems and the role of vision in subterranean rodents: diversity of retinal properties and visual system designs. In: Begall S, Burda $\mathrm{H}$, Schleich CE (eds) Subterranean rodents - news from underground, Springer, Heidelberg, pp 129-160 
Oosthuizen MK (2007) The reproductive biology of the solitary Cape mole-rat, Georychus capensis and the social Natal mole-rat, Cryptomys hottentotus natalensis (Rodentia: Bathyergidae). Doctoral dissertation, University of Pretoria.

O’Riain MJ, Jarvis JUM, Faulkes CG (1996) A dispersive morph in the naked mole-rat. Nature 380:619-621

Roof RL, Stein DG (1999) Gender differences in Morris water maze performance depend on task parameters. Physiol Behav 68:81-86

Sherry DF (1998) The ecology and neurobiology of spatial memory. In: Dukas R (ed) Cognitive Ecology: The evolutionary ecology of information processing and decision making, University of Chicago Press, Chicago and London, pp 261-296

Sichilima AM, Bennett NC, Faulkes CG, Le Comber SC (2008) Evolution of African molerat sociality: burrow architecture, rainfall and foraging in colonies of the cooperatively breeding Fukomys mechowii. J Zool Lond 275:276-282

Skinner JD, Smithers RHN (1990) The mammals of the southern African subregion. University of Pretoria, Pretoria

Sovrano,V. A., Bisazza, A.\&Vallortigara, G. 2003 Modularity as a fish (Xenotoca eiseni ) views it: conjoining geometric and nongeometric information for spatial reorientation. J Exp Psychol Anim Behav Process 29:199-210 
Spinks AC, Jarvis JUM, Bennett NC (2000) Comparative patterns of philopatry and dispersal in two common mole-rat populations: implications for the evolution of mole-rat sociality. J Anim Ecol 69:224-234

Tableman M, Stahel WA (2009) Nonparametric methods for employment termination times with competing causes. Stats Interface 2:37-44

Vallortigara, G. 1996 Learning of colour and position cues in domestic chicks: males are better at position, females at colour. Behav Process 36:289-296

Van Haaren F, Wouters M, Van de Poll NE (1987) Absence of behavioral differences between male and female rats in different radial-maze procedures. Physiol Behav $39: 409-412$

Van Praag H, Kempermann G, Gage FH (2000) Neural consequences of environmental enrichment Nature Rev Neuro 1:191-197

Vleck D (1979) The energy cost of burrowing by the pocket gopher Thomomys bottae. Physiol Zool 52:391-396

Vleck D (1981) Burrow structure and foraging cost in the fossorial rodent Thomomys bottae. Oecologia 49:391-396

White C R (2005) The allometry of burrow geometry J. Zool Lond 265:395-403 
Zelová J, Šumbera R, Okrouhlík J, Burda H (2010) Cost of digging is determined by intrinsic factors rather than by substrate quality in two subterranean rodent species. Physiol Behav 99:54-58 


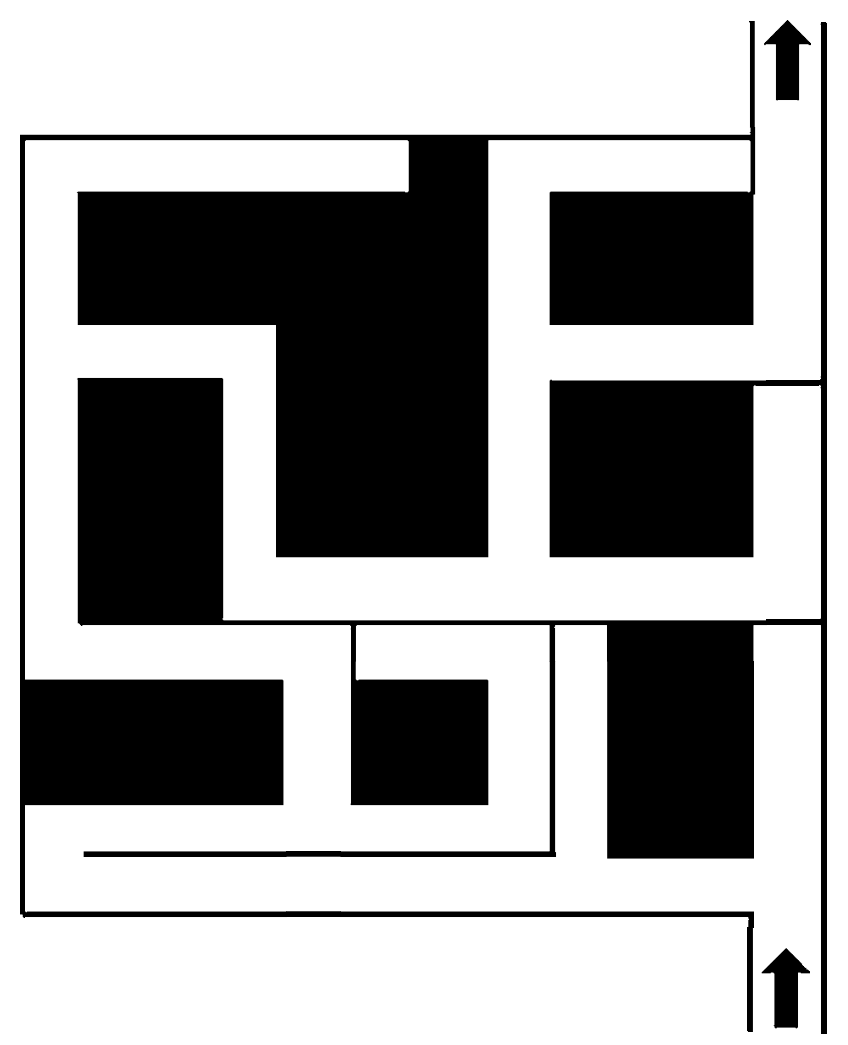

Fig. 1 Maze used to test spatial learning and memory in wild living and captive mole-rats
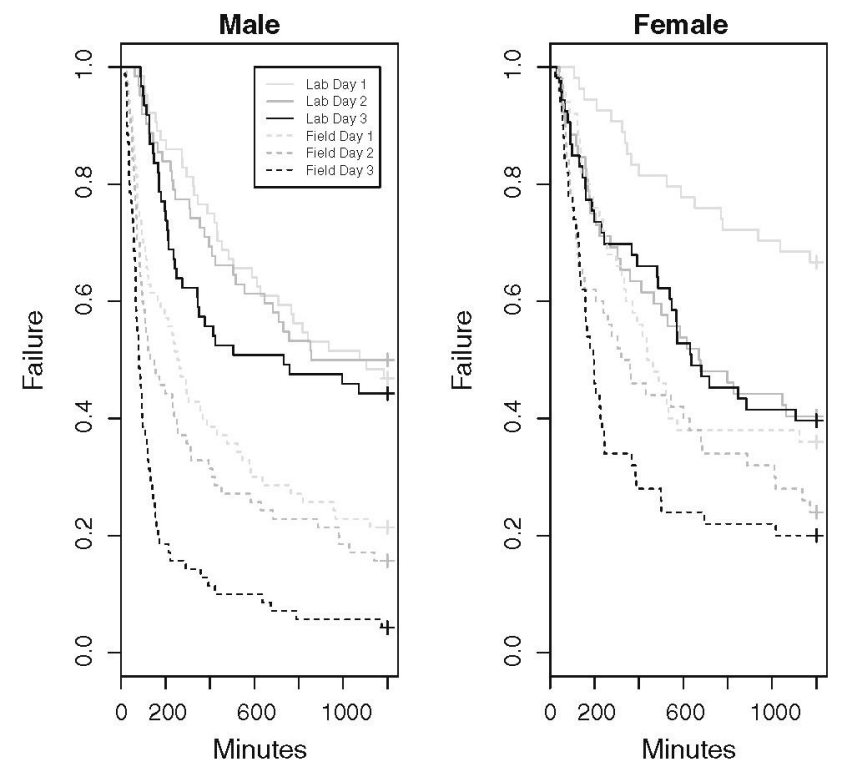

Fig. 2 Hazard plot for completion of maze times. The plot describes the proportion of molerats which are still to complete the maze at each minute into the trial 

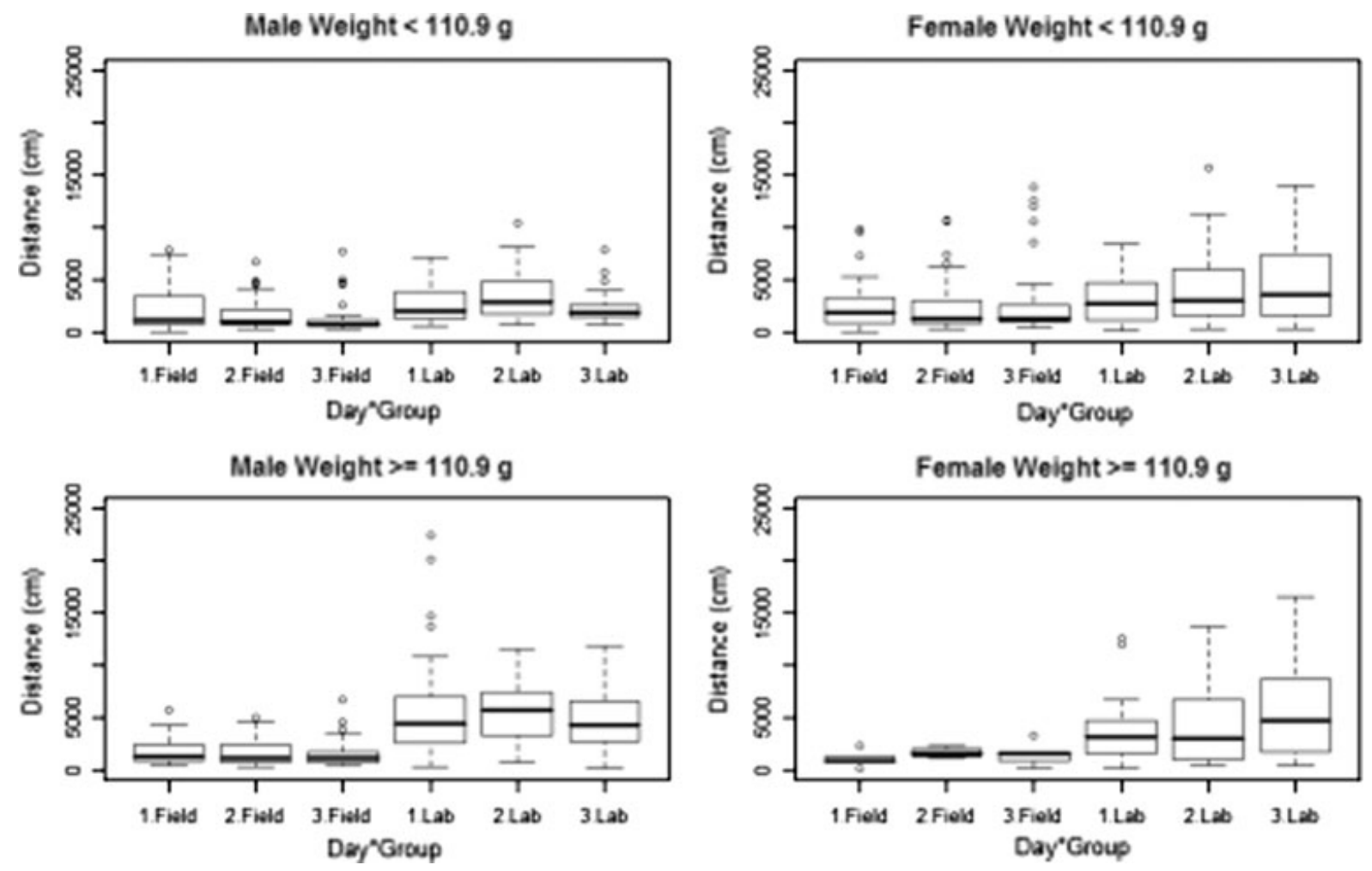

Fig. 3 Box plots of the distance travelled grouped according to sex, 'group' and body mass
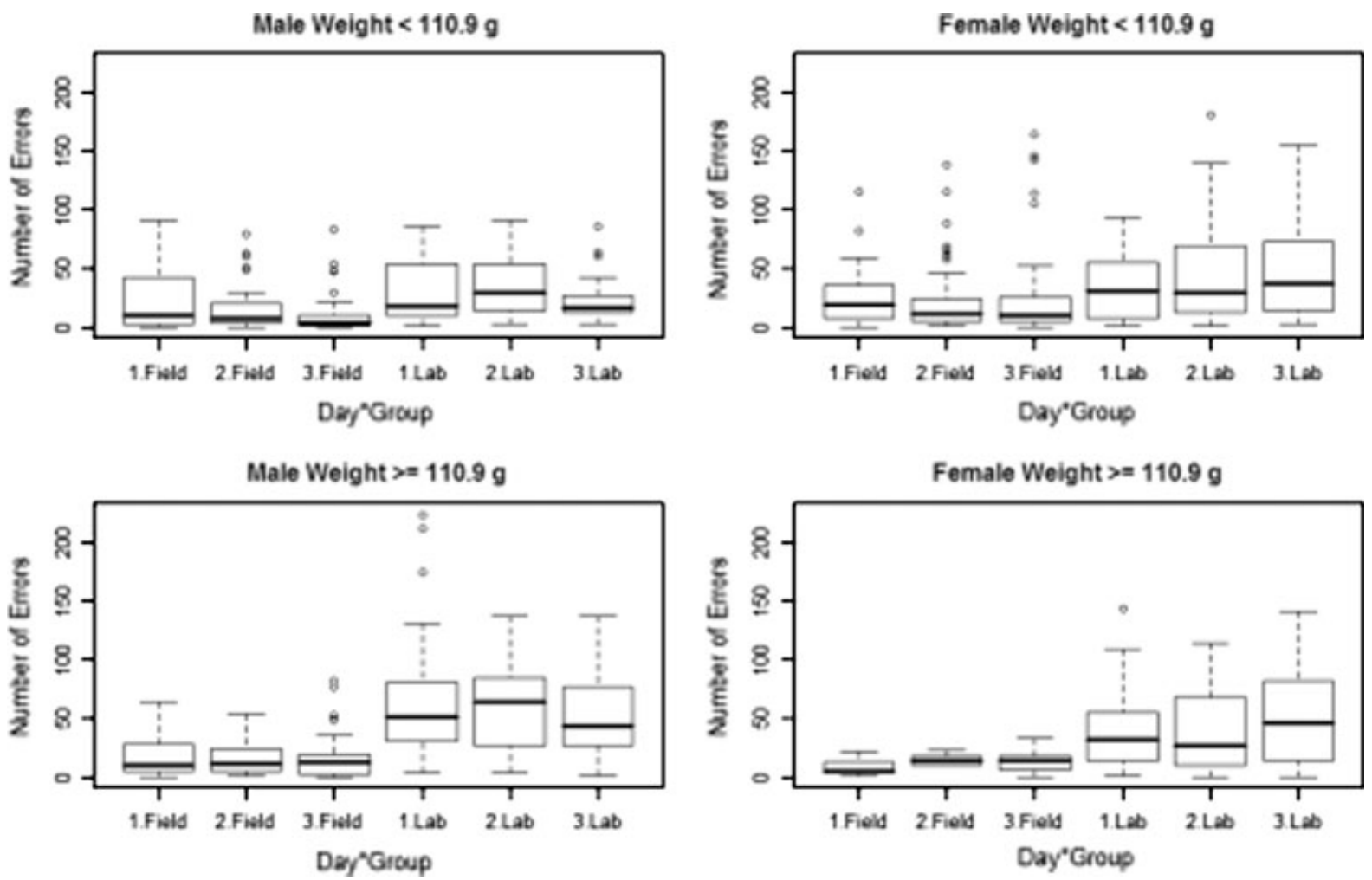

Fig. 4 Box plots of the number of errors grouped according to sex, 'group' and body mass 


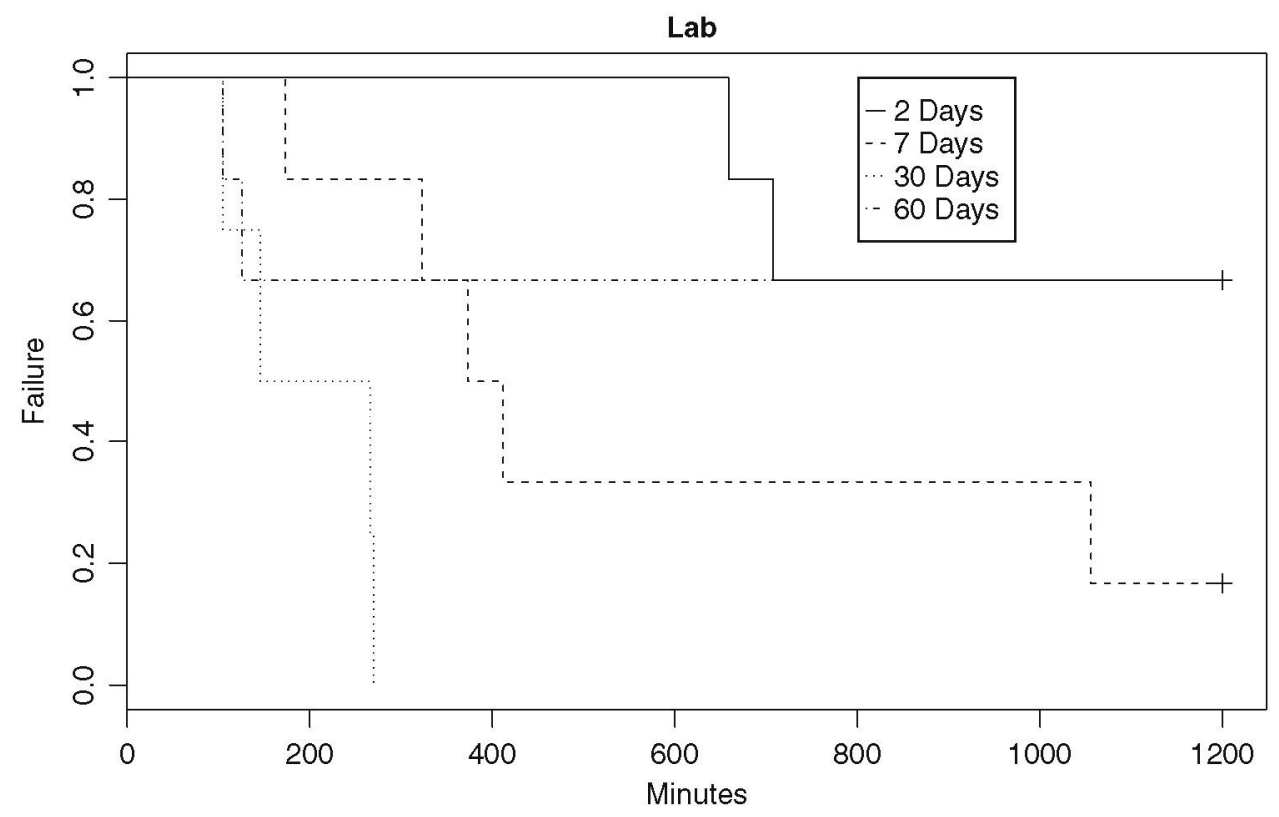

Field

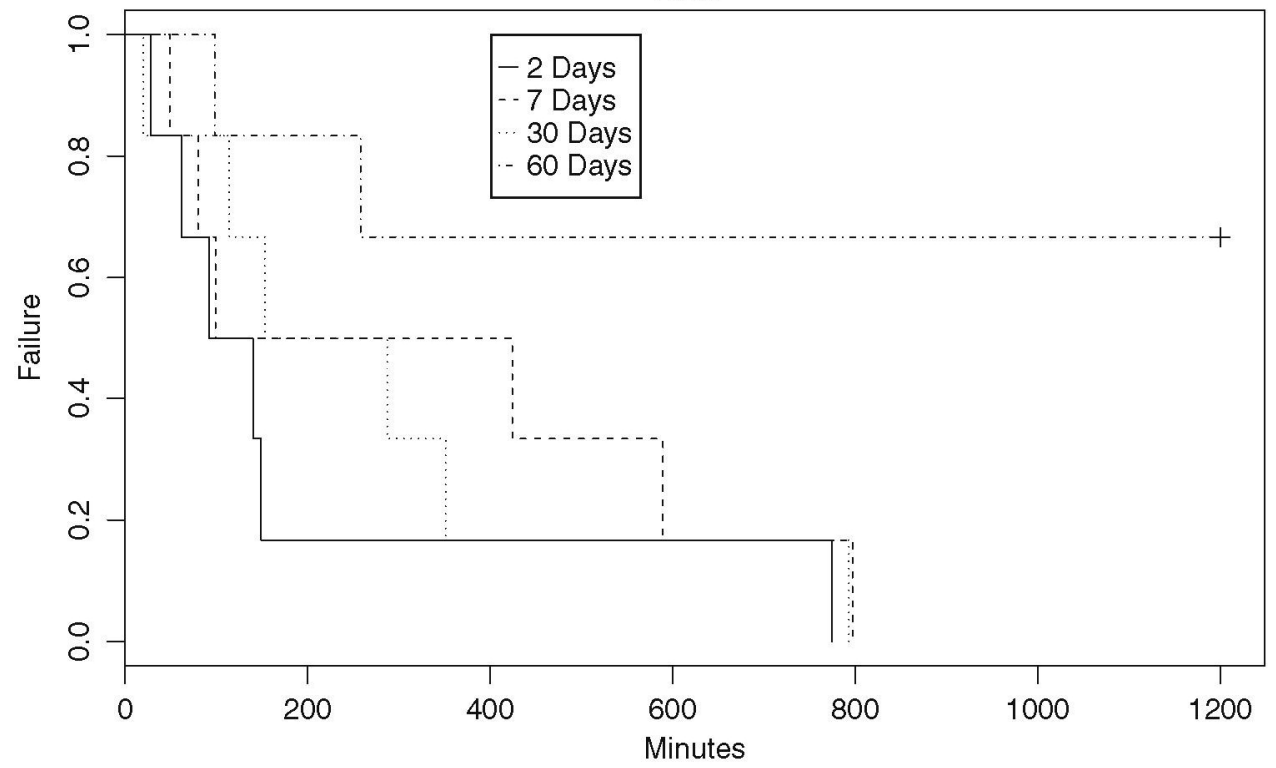

Fig. 5 Plot of hazard functions for the number of days after familiarising the mole-rats with the maze, separated by group 
Table 1 Generalised linear model results for hazard function, modelling the time it takes to complete the maze. $z$-statistics marked $\left(^{*}\right)$ are significant at the $5 \%$ level, $\left({ }^{* *}\right)$ at the $1 \%$ level, and $(* * *)$ at the $0.1 \%$ level.

\begin{tabular}{|c|c|c|c|}
\hline Effect & Coefficient & Standard & z-statistic \\
\hline & Estimate & Error & \\
\hline Intercept & 7.466 & 0.759 & $9.84 * * *$ \\
\hline Group: Lab & 7.768 & 1.380 & $5.63 * * *$ \\
\hline Day: 2 & -2.232 & 0.985 & $-2.27 *$ \\
\hline Day: 3 & -5.064 & 1.001 & $-5.06 * * *$ \\
\hline Sex: Male & -1.070 & 0.889 & -1.20 \\
\hline Weight & -0.007 & 0.009 & -0.75 \\
\hline Group: Lab*Day: 2 & -1.272 & 0.675 & -1.88 \\
\hline Group: Lab*Day: 3 & -1.751 & 0.671 & $-2.61 * *$ \\
\hline Group: Lab*Sex: Male & -10.972 & 1.617 & $-6.79 * * *$ \\
\hline Day: $2 *$ Sex: Male & 0.649 & 1.172 & 0.55 \\
\hline Day: 3*Sex: Male & 2.104 & 1.176 & 1.79 \\
\hline Day: $2 *$ Weight & 0.023 & 0.012 & $1.98 *$ \\
\hline Day: $3 *$ Weight & 0.051 & 0.012 & $4.30 * * *$ \\
\hline Sex: Male*Weight & 0.006 & 0.001 & 0.63 \\
\hline Group: Lab*Weight & -0.054 & 0.011 & $-4.89 * * *$ \\
\hline Group: Lab*Day: 2*Sex: Male & 1.543 & 0.770 & $2.00 *$ \\
\hline Group: Lab*Day: 3*Sex: Male & 2.660 & 0.762 & $3.49 * * *$ \\
\hline Day: 2*Sex: Male*Weight & -0.011 & 0.013 & -0.82 \\
\hline Day: 3*Sex: Male*Weight & -0.036 & 0.013 & $-2.65 * *$ \\
\hline
\end{tabular}


Table 2 Coefficient estimates from linear model for the distance travelled by the mole-rat in the maze. $t$-statistics marked $(*)$ are significant at the $5 \%$ level, $\left({ }^{* *}\right)$ at the $1 \%$ level, and $(* * *)$ at the 0.1\% level. Residual degrees of freedom are 658.

\begin{tabular}{|c|c|c|c|}
\hline Effect & Coefficient & Standard & $t$-statistic \\
\hline & Estimate & Error & \\
\hline Intercept & 658.133 & 1156.900 & 0.57 \\
\hline Day: 2 & -481.784 & 1135.061 & -0.42 \\
\hline Day: 3 & -1041.365 & 1135.059 & -0.92 \\
\hline Sex: Male & 1637.592 & 1168.112 & 1.40 \\
\hline Group: Lab & -2904.452 & 2340.113 & -1.24 \\
\hline Weight & 21.187 & 13.273 & 1.60 \\
\hline Day 2:Sex: Male & -312.338 & 545.623 & -0.57 \\
\hline Day3: Sex: Male & -1395.599 & 544.829 & $-2.56 *$ \\
\hline Group: Lab*Day: 2 & 7601.065 & 2383.482 & $3.19 * *$ \\
\hline Group: Lab*Day: 3 & 9403.531 & 2393.748 & $3.92 * * *$ \\
\hline Group: Lab*Sex: Male & -7165.066 & 2261.977 & $-3.17 * *$ \\
\hline Day: 2*Weight & 4.453 & 11.887 & 0.38 \\
\hline Day: 3*Weight & 16.315 & 11.886 & 1.37 \\
\hline Sex: Male*Weight & -22.649 & 12.644 & -1.80 \\
\hline Group: Lab*Weight & 29.533 & 21.928 & 1.35 \\
\hline Group: Lab*Day: 2*Sex: Male & -57.939 & 20.813 & $-2.78 * *$ \\
\hline Group: Lab*Day: 3*Sex: Male & 76.617 & 20.923 & $-3.66 * * *$ \\
\hline Group: Lab*Sex: Male*Weight & 71.259 & 21.053 & $3.39 * * *$ \\
\hline
\end{tabular}


Table 3 Coefficient estimates from linear model for the number of errors made by the mole-rat in the maze. $t$-statistics marked $(*)$ are significant at the $5 \%$ level, $\left({ }^{* *}\right)$ at the $1 \%$ level, and $(* * *)$ at the $0.1 \%$ level. Residual degrees of freedom are 658 .

\begin{tabular}{|c|c|c|c|}
\hline \multirow{2}{*}{ Effect } & \multirow{2}{*}{$\begin{array}{l}\text { Coefficient } \\
\text { Estimate }\end{array}$} & \multirow{2}{*}{$\begin{array}{l}\text { Standard } \\
\text { Error }\end{array}$} & \multirow{2}{*}{ t-statistic } \\
\hline & & & \\
\hline Intercept & 0.909 & 13.086 & 0.07 \\
\hline Day: 2 & -5.714 & 12.839 & -0.45 \\
\hline Day: 3 & -16.369 & 12.839 & -1.28 \\
\hline Sex: Male & 17.118 & 13.213 & 1.30 \\
\hline Group: Lab & -1.266 & 26.470 & -0.05 \\
\hline Weight & 0.296 & 0.1501 & $1.97 *$ \\
\hline Day 2: Sex: Male & -0.722 & 6.172 & -0.12 \\
\hline Day3: Sex: Male & -14.055 & 6.163 & $-2.28 *$ \\
\hline Group: Lab*Day: 2 & 81.946 & 26.960 & $3.04 * *$ \\
\hline Group: Lab*Day: 3 & 109.937 & 27.076 & $4.06 * * *$ \\
\hline Group: Lab*Sex: Male & -106.995 & 25.586 & $-4.18 * * *$ \\
\hline Day: 2*Weight & 0.0319 & 0.134 & 0.24 \\
\hline Day: 3*Weight & 0.225 & 0.134 & 1.67 \\
\hline Sex: Male*Weight & -0.269 & 0.143 & -1.88 \\
\hline Group: Lab*Weight & 0.035 & 0.248 & 0.14 \\
\hline Group: Lab*Day: $2 *$ Sex: Male & -0.628 & 0.235 & $-2.67 * *$ \\
\hline Group: Lab*Day: 3*Sex: Male & -0.934 & 0.237 & $-3.95 * * *$ \\
\hline Group: Lab*Sex: Male*Weight & 1.073 & 0.238 & $4.50 * * *$ \\
\hline
\end{tabular}


Table 4 Generalised linear model results for hazard function, modelling the time it takes to complete the maze. $z$-statistics marked $\left(^{*}\right)$ are significant at the $5 \%$ level, $\left({ }^{* *}\right)$ at the $1 \%$ level, and $(* * *)$ at the $0.1 \%$ level.

\begin{tabular}{|c|c|c|c|}
\hline Effect & Coefficient & Standard & z-statistic \\
\hline & Estimate & Error & \\
\hline Intercept & 5.729 & 1.005 & $5.70 * * *$ \\
\hline Day: 7 & 2.494 & 0.932 & $2.68 * *$ \\
\hline Day: 30 & 1.153 & 0.690 & 1.67 \\
\hline Day: 60 & 3.332 & 0.872 & $3.82 * * *$ \\
\hline Sex: Male & -2.761 & 1.374 & $-2.01 *$ \\
\hline Group: Lab & 3.331 & 0.944 & $3.53 * * *$ \\
\hline Weight & -0.018 & 0.013 & -1.40 \\
\hline Day 7: Sex: Male & -1.904 & 0.940 & $-2.03 *$ \\
\hline Day30: Sex: Male & -2.444 & 0.874 & $-2.80 * *$ \\
\hline Day60: Sex: Male & -2.326 & 1.290 & -1.80 \\
\hline Group: Lab*Day: 7 & -3.675 & 1.119 & $-3.29 * *$ \\
\hline Group: Lab*Day: 30 & -2.665 & 0.980 & $-2.72 * *$ \\
\hline Group: Lab*Day: 60 & -3.440 & 1.149 & $-2.99 * *$ \\
\hline Sex: Male*Weight & 0.047 & 0.016 & $3.02 * *$ \\
\hline
\end{tabular}

